This is the author's manuscript for publication. The publisher-formatted version may be available through the publisher's web site or your institution's library.

\title{
Mapping resistance to the bird cherry-oat aphid and the greenbug in wheat using sequence-based genotyping
}

\author{
L. A. Crespo-Herrera, Eduard D. Akhunov, L. Garkava-Gustavsson, Katherine W. \\ Jordan, C. Michael Smith, R. P. Singh, I. Åhman
}

\section{How to cite this manuscript}

If you make reference to this version of the manuscript, use the following information:

Crespo-Herrera, L. A., Akhunov, E., Garkava-Gustavsson, L., Jordan, K. W., Smith, C. M., Singh, R. P., \& Åhman, I. (2014). Mapping resistance to the bird cherry-oat aphid and the greenbug in wheat using sequence-based genotyping.

\section{Published Version Information}

\section{Citation:}

Crespo-Herrera, L. A., Akhunov, E., Garkava-Gustavsson, L., Jordan, K. W., Smith, C. M., Singh, R. P., \& Åhman, I. (2014). Mapping resistance to the bird cherry-oat aphid and the greenbug in wheat using sequence-based genotyping. Theoretical and Applied Genetics, 127(9), 1963-1973.

Digital Object Identifier (DOI): 10.1007/s00122-014-2352-5

Publisher's Link: http://link.springer.com/article/10.1007/s00122-014-2352-5

This item was retrieved from the K-State Research Exchange (K-REx), the institutional repository of Kansas State University. K-REx is available at http://krex.ksu.edu 


\title{
Mapping resistance to the bird cherry-oat aphid and the greenbug in wheat using sequence-based genotyping
}

Theoretical and Applied Genetics, 127(9), 1963-1973.

Crespo-Herrera L. A. ${ }^{1}$, Akhunov E. ${ }^{2}$, Garkava-Gustavsson L. ${ }^{1}$, Jordan K. ${ }^{2}$, Smith C. M. ${ }^{3}$, Singh R. P. ${ }^{4}$, Åhman I. ${ }^{1}$

${ }^{1}$ Department of Plant Breeding, Swedish University of Agricultural Sciences, P.O. Box 101, SE 23053 Alnarp, Sweden; ${ }^{2}$ Department of Plant Pathology, Kansas State University, Manhattan KS 66506-5502; ${ }^{3}$ Department of Entomology, Kansas State University, Manhattan KS 66506-5502; ${ }^{4}$ International Maize and Wheat Improvement Center (CIMMYT), Apdo. Postal 6-641, 06600, Mexico DF, Mexico

Email: leo.crespo@slu.se

\begin{abstract}
The aphids Rhopalosiphum padi and Schizaphis graminum are important pests of common wheat (Triticum aestivum L.). Characterization of the genetic bases of resistance sources is crucial to facilitate the development of resistant wheat cultivars to these insects. We examined 140 recombinant inbred lines (RILs) from the cross of the susceptible wheat Seri M82 with the synthetic hexaploid wheat CWI76364, resistant to both aphid species. The RILs were phenotyped for $R$. padi antibiosis and tolerance traits. Phenotyping of $S$. graminum resistance was based on leaf chlorosis in a greenhouse screening, and also on the number of $S$. graminum per tiller in a field trial. Seedling pubescence was scored in each RIL. Using a sequence-based genotyping method we located genomic regions associated to these resistance traits. One QTL for R. padi antibiosis was found in chromosome 4BL; it explained $10.2 \%$ of phenotypic variation and was located $14.6 \mathrm{cM}$ apart from the pubescence locus. However, we did not find any association between plant pubescence and the other resistance traits. We found two QTLs for tolerance to $R$. padi in chromosomes 5AL and 5BL, with an epistatic interaction between a locus in chromosome $3 \mathrm{AL}$ and the tolerance QTL in 5AL. These genomic regions together explained about 35\% of the phenotypic variation. We confirmed the location of a previously reported gene for $S$. graminum resistance $(G b a)$ in 7DL and found an additional, novel QTL associated with the number of aphids per tiller in chromosome 2DL. This is the first report where resistance to R. padi in wheat is mapped and also where chromosome 2DL shown to be associated with $S$. graminum resistance.
\end{abstract}

Key words: Rhopalosiphum padi, Schizaphis graminum, synthetic hexaploid wheat, genotyping by sequencing, resistance, QTLs.

\section{INTRODUCTION}

The aphids Rhopalosiphum padi L. and Schizaphis graminum (Rondani) are two of the most serious pests of wheat (Triticum aestivum L.), and can significantly reduce wheat yields by $30-40 \%$ solely due to feeding and up to $60 \%$ when such damage is combined with virus infection (Kieckhefer and Gellner 1992; Voss et al. 1997; Riedell et al. 2003). Both aphid species are widely distributed in regions where wheat is grown (Blackman and Eastop 2007), however, S. graminum is not a wheat pest in northern Europe. One way of contributing to reduce the damage caused by these pests is by breeding resistant varieties.

Including resistance to these aphids as an additional component in conventional wheat breeding programs is challenging. Phenotypic selection for aphid resistance is difficult to apply without sacrificing other breeding goals. It 
requires considerable logistic efforts to maintain large segregating populations in various generations during selection under homogenous aphid pressures across time and space. However, the identification of molecular markers associated with aphid resistance/tolerance genes can greatly facilitate the addition of this component into wheat breeding programs. Therefore, it is crucial to characterize and understand the genetic bases of aphid resistance in wheat germplasm.

Resistance to insects is generally classified as antibiosis, antixenosis and tolerance (Smith 2005). Antibiosis builds on plant characteristics that negatively affect insect physiology; causing higher mortality rates, longer development periods, reduced weight gain, etc. Antixenosis is the reduced suitability of a plant to serve as a host for feeding and reproduction via plant traits that influence the insect host-selection behavior. Tolerance is the ability of a plant to withstand or recover from insect damage without compromising insect behavior or physiology. All three components of insect resistance tend to be present in resistant plants to a certain level. However, oftentimes there is one component that predominates over the others making a plant genotype more antibiotic, antixenotic or tolerant. Some complications at the categorization of insect resistance are, 1) that antixenosis expressed as lower feeding rate may reduce insect performance and then be interpreted as antibiosis and 2) that tolerance measured as reduced insectspecific plant symptoms may be due to fewer or less of insect feeding and not due to a plant response. In the present study, we measured weight gain of $R$. padi nymphs after a certain feeding period to estimate antibiosis and reduced seedling biomass under $R$. padi feeding pressure to estimate tolerance. Furthermore, we scored leaf chlorosis as an estimate of plant tolerance to $S$. graminum and measured $S$. graminum density in a field trial to estimate antixenosis/antibiosis.

The wild relatives of wheat are rich sources of genetic variation that may confer resistance to various biotic stresses. One way to incorporate such variation in bread wheat is by synthesizing hexaploid wheats (SHWs) from the cross of a tetraploid species with Aegilops tauschii Coss. Several resistance traits to various pests and diseases have been reported in SHWs, and in some cases they have been successfully deployed into commercial varieties (Ogbonnaya et al. 2013). For instance in the case of aphids, Smith and Starkey (2003), evaluated a large set of SHWs for resistance to $S$. graminum and found high levels of variation that was further characterized by Zhu et al. (2005).

Efforts to deploy resistance to S. graminum in wheat were made since the 1950's (Porter et al. 1997; Berzonsky et al. 2003) and this has led to the identification of several resistance genes. Currently there are 14 major genes for resistance to S. graminum in wheat that have been identified (McIntosh et al. 2010; Crespo-Herrera 2012). Of those, one is from Triticum turgidum L., two from Secale cereale L., one from T. aestivum, one from Aegilops speltoides Tausch, and 9 from A. tauschii. Some of these genes have been incorporated into wheat cultivars, for instance the gene Gb3 from A. tauschii (Ogbonnaya et al. 2013). There are several S. graminum biotypes that differ in their virulence patterns to these resistance genes; biotypes $\mathrm{E}$ and I are the most common on wheat in the USA (Burd and Porter 2006).

Despite that $R$. padi is considered to be an important pest in wheat, there are no resistant cultivars developed for this aphid species. This may be because $R$. padi damage is not as evident as for other aphid species; for instance $S$. graminum, which does cause clear plant symptoms and so the selection of resistant-plant progeny can be based on that trait. Additionally, the polyphagy and wide host adaptation of $R$. padi can make it difficult to find resistance sources with adequate protection levels. Possibly this is why there are no published studies on the genetics of $R$. padi resistance in wheat, and so far no aphid biotypes of this species have been reported either. However, resistance to this species has been mapped in barley, and a quantitative trait locus (QTL) in chromosome $3 \mathrm{H}$ that explained $9 \%$ of the aphid growth variation was reported by Cheung et al. (2010).

Next generation sequencing technologies provide great opportunities to unravel the genetic bases of quantitative traits by supplying large amounts of data in a cost and time effective manner. Genotyping by sequencing (GBS) is one of the applications. In the development of GBS libraries the genome complexity is reduced by the use of methylation-sensitive restriction enzymes and in combination with multiplex sequencing it is possible to genotype 
entire populations efficiently (Elshire et al. 2011; Poland et al. 2012). This is particularly useful for species with large genomes such as wheat, where sequencing needs to target non-duplicated regions of the genome in order to produce high quality maps (Spindel et al. 2013). GBS methods can be applied in bi-parental populations to locate genomic regions associated to various agronomic traits of interest (Saintenac et al. 2013).

In this study we identified genomic regions associated to various traits related to resistance against $R$. padi and $S$. graminum in a SHW by using sequencing-based genotyping methods. This is the first time that antibiosis and tolerance to $R$. padi wasn mapped in wheat and also that resistance to $S$. graminum was found in chromosome 2DL.

\section{MATERIALS AND METHODS}

\section{Plant material}

We developed a mapping population of 140 F6 recombinant inbred lines (RILs) with the single-head descent method at the International Maize and Wheat Improvement Center (CIMMYT) in Mexico. The progenitors of the population were the spring wheat Seri M82 (aphid susceptible) and the SHW CWI76364 (aphid resistant). The pedigree of CWI76364 is: Triticum dicoccum PI 94623/A. tauschii (1027) (Lage et al. 2003). This A. tauschii accession was also progenitor of the population where the Gba resistance gene was mapped by Zhu et al. (2005).

Phenotyping and statistical analyses of phenotypic data

The 140 RILs were phenotyped for resistance to R. padi and S. graminum. Evaluations of R. padi resistance were conducted in the Department of Plant Breeding at the Swedish University of Agricultural Sciences (SLU). $S$. graminum resistance evaluations were performed in the Department of Entomology at the Kansas State University (KSU) and also at CIMMYT's breeding station in Ciudad Obregon, Mexico.

All statistical analyses of the phenotypic data were made with SAS statistical software v9.3 (SAS Institute Inc 2011).

\section{Rhopalosiphum padi phenotyping}

Antibiosis and tolerance were evaluated with virus-free individuals of $R$. padi reared on oat plants under greenhouse conditions at ca. $22^{\circ} \mathrm{C}$, with a minimum of $16 \mathrm{~h}$ of light, supplemented when needed with $400 \mathrm{~W}$ high-pressure sodium lamps.

Antibiosis evaluations started by placing eight seeds of each RIL on moist filter paper in Petri dishes. Dishes were kept in a refrigerator at $5^{\circ} \mathrm{C}$ during 3 days and thereafter for 2 days at room temperature. After that period four germinated seeds were singly transplanted in $10 \mathrm{~cm}$ diameter plastic pots $(300 \mathrm{ml})$ filled with Weibull's Kronmull ${ }^{\circledR}$ potting soil with Leca. Seedlings were grown in a walk-in climate controlled chamber at $22^{\circ} \mathrm{C}, 80 \% \mathrm{RH}$ and $16 \mathrm{~h}$ light at the intensity of $250 \mu \mathrm{mol}$ photons $\mathrm{m}^{-2} \mathrm{~s}^{-1}$ at plant level. The RILs were tested in seven time-separated incomplete blocks with a maximum of 24 lines per incomplete block including both parents. Each line was replicated four times within each incomplete block.

When plants reached 2-3 leaf stage they were individually exposed to five $1^{\text {st }}$ instar nymphs that were born within a period of 24 hours. These new-born nymphs were obtained from alate individuals previously caged on oat plants for 24 hours. The new-born nymphs were confined at the plant base with transparent cylindrical acrylic cages $(2 \mathrm{~cm}$ diameter, $5 \mathrm{~cm}$ length), sealed with cotton wool at the bottom and the top. Four days after infestation aphids were individually weighted on a microbalance (Mettler M3). Analysis of variance and least-square means were estimated using the MIXED procedure in SAS, with RILs and replications nested within incomplete blocks. Variance 
components were estimated to calculate heritability. For the QTL analysis, the mean aphid weight on each RIL was expressed as the proportion of the mean aphid weight displayed for the susceptible parent.

Tolerance of each RIL was measured as biomass reduction in an augmented split plot designed experiment with two treatments, Infested (1) and Non-infested (2). Each parent of the population was repeated 15 times. Biomass reduction was calculated with the formula: 1-(I/NI), where I is equal to the plant biomass of the aphid-infested RIL, and NI is equal to the plant biomass of the same non-infested RIL. The experiment was conducted under the same greenhouse conditions as for $R$. padi rearing, using the same type of soil as in the antibiosis tests. First, four seeds of each RIL were sown in flats $(41 \mathrm{~cm} \times 61 \mathrm{~cm} \times 11 \mathrm{~cm})$. Three days after germination, excess plants were gently pulled out to allow only one plant to grow, and to select plants of about the same size for the application of treatments. Seedlings of each treatment were grown in separate flats respectively. Plants of treatment 1 were infested every second day with aphids at an approximate density of 45 aphids/plant. Infestations started when seedlings were at 2-3 leaf stage. Plants of treatment 2 were treated with the systemic insecticide Confidor $\mathrm{WG}^{\circledR}$ (Bayer CropScience) at a dose of $3.5 \%$ of active ingredient by pouring the solution in the soil (Dunn et al. 2007). Fifteen days later, plants of both treatments were cut at the soil level and dried during 72 hours at $70^{\circ} \mathrm{C}$. Plants were then weighted on an analytical balance (Sartorius ME215P). Analysis of variance was performed with the MIXED procedure of SAS. Parents and treatments were fixed effects, whereas the RILs and their interaction with the treatments were treated as random effects in the model. Variance components were calculated to estimate heritability.

\section{Schizaphis graminum phenotyping}

Evaluations of $S$. graminum resistance consisted of two separate tests, one testing for leaf symptoms and another for population build up in the field. Screening for leaf chlorosis was performed under greenhouse conditions at ca. $22^{\circ} \mathrm{C}$, $80 \% \mathrm{RH}$ and $16 \mathrm{~h}$ light at KSU. Virus-free starter colonies of $\mathrm{S}$. graminum biotype E were obtained from USDA-

ARS and maintained on Jagger wheat. Biotype $\mathrm{E}$ is common and virulent to wheat carrying resistance genes $G b 1$ and Gb2 (Burd and Porter 2006) but not to the other 12 known resistance genes (Crespo-Herrera 2012). Resistance was scored as R (resistant) or S (susceptible) based on leaf chlorosis symptoms in the same fashion as by Zhu et al. (2005). The entries of the population were sown in hill plots of 6-8 seeds/hill in a complete randomized block design with four replicates. Plants were infested with an approximate density of 5 aphids/hill plot at 2-3 leaf stage. Resistance was scored when the susceptible parent showed more than $50 \%$ chlorosis. Statistical analysis of R/S responses was made with the LOGISTIC procedure of SAS.

Outbreaks of S. graminum commonly occur in Northwestern Mexico during the wheat growing season where we performed the field evaluation. The field test was conducted at CIMMYT's Ciudad Obregon experimental station, in Mexico ( $\left.27^{\circ} 37^{\prime} \mathrm{N}, 109^{\circ} 93^{\prime} \mathrm{W}\right)$ during the winter season 2012-2013. The 140 RILs were sown on December $20^{\text {th }}$, 2012. The experiment was laid out in a rectangular row-column augmented design (6x30), with the population's parents replicated 12 times. Eight to ten seeds of each RIL where planted in a $10 \mathrm{~cm}$ hill plot in a bed-planting system with $0.8 \mathrm{~m}$ of distance between beds and $1 \mathrm{~m}$ distance between hill plots along the rows. The trial was irrigated six times throughout the crop season by surface irrigation. Fertilization rate was 200-50 (N-P), of which 50-50 was applied at sowing, and 150-00 was applied 3-4 weeks after sowing along with the first irrigation. Weeds were controlled manually. The number of aphids in ten tillers was counted in each hill plot when S. graminum reached its highest population density in a neighboring trial. The number of aphids/tiller was transformed to a logarithmic scale before the statistical analysis. The MIXED procedure of SAS was used to make the analysis of variance. Parents were fixed effects in the model, whereas the RILs were treated as random effects. Variance components were used for the estimation of heritability.

Pubescence phenotyping 
Seedlings of the mapping population were grown in a greenhouse at CIMMYT's headquarters $\left(19^{\circ} 32^{\prime} \mathrm{N}, 98^{\circ} 50^{\prime}\right.$ W) in October 2012 at an average temperature of $25^{\circ} \mathrm{C}$ and natural day-length conditions. Leaf pubescence was scored as either present or absent when the plants had reached 2-3 leaf stage.

Genotyping

Seedlings of the 140 RILs and the parents were grown in a greenhouse in the Department of Plant Pathology at the Kansas State University. Plant tissue was collected when plants reached 2-4 leaf stage. Extractions of DNA were made with a QUIAGEN DNeasy 96 Plant Kit $^{\circledR}$, by following the manufacturer instructions. DNA was quantified using the Quanti-iT ${ }^{\mathrm{TM}}$ PicoGreen ${ }^{\circledR}$ and concentrations were then normalized to $20 \mathrm{ng} / \mu \mathrm{l}$.

Genotyping by sequencing (GBS) libraries were obtained following the protocol reported by Saintenac et al. (2013). The combination of PstI (barcoded adapter) and MseI (Y-adapter) restriction enzymes was used. We used a set of 96 barcodes with sticky ends complementary to the 3' overhang of PstI and MseI-Y-adapters (Saintenac et al. 2013). Prior to the use of the adapters, the common primer (5'-CTCGGCATTCCTGCTGAACCGCTCTTCCGATCT-3') was annealed with the enzyme specific primer of MseI (5'-taAGATCGGAAGAGCGGGGACTTTAAGC-3') to prepare the Y-adapters. Annealing was done with a thermal cycler in $10 \mathrm{nM}$ Tris- $\mathrm{HCl}$ and $50 \mathrm{nM} \mathrm{NaCl}$ buffer. Temperature was reduced from $95^{\circ} \mathrm{C}$ to $30^{\circ} \mathrm{C}$ in 65 cycles at a rate of $1^{\circ} \mathrm{C} / \mathrm{min}$. Barcode adapters were then adjusted to a concentration of $0.2 \mu \mathrm{M}$.

DNA samples were pipetted in two 96-well plates of 72 and 70 samples each. Restriction, ligation and amplification processes were performed to construct the genomic libraries (Poland et al. 2012; Saintenac et al. 2013). Restriction of DNA was made with a restriction mix that consisted of $20 \mu 1$ of each sample, 3.0 $\mu 1$ of NEB T4 DNA ligase buffer 3, $0.5 \mu 1$ of PstI (10 units), $1 \mu \mathrm{l}$ of MseI (10 units) and 5.5 $\mu 1$ of $\mathrm{H}_{2} \mathrm{O}$. Ligation was performed by adding to the restriction digest $(30 \mu \mathrm{l})$ of each sample, $3 \mu \mathrm{l}$ of the unique barcode adapter, $8 \mu 1$ of the Y-adapter and $9 \mu 1$ of ligation NEB Master Mix ( $2 \mu 1$ of T4 DNA ligase buffer, $0.5 \mu 1$ T4 DNA ligase and $6.5 \mu 1$ water). Samples were then incubated at $22^{\circ} \mathrm{C}$ for two hours and maintained at $65^{\circ} \mathrm{C}$ for 20 minutes to inactivate the T4 DNA ligase. Before the amplification step, the samples were purified with a QIAGEN QIAquick PCR Purification $\mathrm{Kit}^{\circledR}$. Then $5 \mu 1$ of each

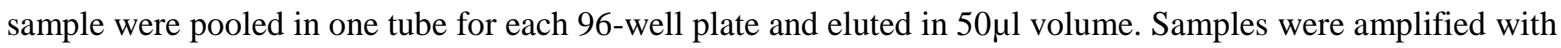
$10 \mu \mathrm{M}$ of PCR primers 5 AATGATACGGCGACCACCGAGATCTACACTCTTTCCCTACACGACGCTCTTCCGATCT-3' (forward) and 5'- CAAGCAGAAGACGGCATACGAGATCGGTCTCGGCATTCCTGCTGAA-3' (reverse) using a Taq 2x Master Mix from NEB. There were 18 PCR cycles of $95^{\circ} \mathrm{C}(30 \mathrm{~s}), 62^{\circ} \mathrm{C}(10 \mathrm{~s}), 68^{\circ} \mathrm{C}(20 \mathrm{~s})$ that were terminated with a final extension at $72^{\circ} \mathrm{C}$ for 5 minutes. The size and distribution of the DNA fragments in the genomic libraries were analyzed with the Bioanalyzer Aligen DNA 1000. Libraries were sequenced in an Illumina HiSeq2000 flow-cell machine.

SNP calling and QTL analysis.

We processed the GBS reads for SNP calling with the Universal Network Enabled Analysis Kit (UNEAK) implemented in TASSEL 3.0 standalone version (Lu et al. 2013). The GBS raw data from the Illumina HiSeq2000 machine were first trimmed to a length of $64 \mathrm{bp}$ to keep high quality sequences, then identical reads were grouped into tags; further these tags were pairwise aligned to identify single base pair mismatches, which represented candidate SNPs. Each pairwise alignment represents a node in a network; the complex networks and the ones without a single reciprocal mismatch were discarded with a network filter at an error rate tolerance of 0.03 to end up only with the reciprocal tag pairs that were used for SNP calling. After processing the Ilumina Fastq files with the UNEAK pipeline, a total of 1313 GBS markers were used for the QTL analysis.

We made the linkage groups with the 1313 GBS markers, and also with the categorical responses of each RIL to $S$. graminum R/S leaf symptoms and pubescence present/absent scores. We used the software ICIMapping (Li et al. 2007) with a LOD score of 9 for grouping the markers, the Traveling Salesman algorithm for ordering, and a 
window size of $5 \mathrm{cM}$ for rippling the linkage groups. Linkage groups having less than three markers or markers with no linkage where discarded from the analysis (altogether three out of 25). For assigning chromosome groups to the genomic regions of interests, $20 \%$ of the marker sequences in each LG were systematically searched with the Basic Local Alignment Search Tool (BLAST) in the wheat genome sequence published by Brenchley et al. (2012) and available at EnsamblPlants (http://plants.ensembl.org/Triticum_aestivum/Info/Index).

The program ICIMapping was also used to perform inclusive-composite interval QTL mapping. Significance threshold for the $10 \%$ tail of null distribution was obtained with a run of 1000 permutations and a 0.001 probability value for markers to enter into the QTL model (Da Costa E Silva et al. 2012a; Da Costa E Silva et al. 2012b). Linkage groups and QTLs were plotted with MapChart software (Voorrips 2002).

Additionally, to determine if QTLs associated to R. padi tolerance were related to plant growth per se, we conducted a multi-environmental QTL analysis with ICIMapping, where treatments Infested and Non-infested were considered as different environments.

To identify pairs of interacting loci we made a 2-dimensional 2-QTL scan with the R/qtl package in the R software v3.0.1 (Broman et al. 2003; R core team 2013). The conditional genotype probabilities of the markers were first calculated with the "calc.genoprob" instruction. The LOD score of the model including an epistatic term and the LOD score of the model accounting only for additive effects were obtained with the "scantwo" instruction. A run of 1000 permutations was made to set the threshold of the $5 \%$ tail of the null distribution. If interactions between loci were identified with the two-dimensional scan, then the positions of the main QTLs and the epistatic loci were used to estimate the effect of interactions in a multiple interval mapping framework in the R/qtl package.

A further analysis of interacting markers was made by using the MIXED procedure in SAS. We calculated leastsquare means for the phenotypes of the groups of genotype combinations given by the markers linked to the genomic regions of interest, and used Tukey's test for comparisons of means to assess the difference among phenotype values given by the marker genotypes.

\section{RESULTS}

There were 22 linkage groups (LGs) used for the analysis, spanning a total length of $2252.7 \mathrm{cM}$ based on 1309 GBS markers. Thus, there was on average one marker every $1.72 \mathrm{cM}$. The BLAST analysis of the GBS sequences showed that all 21 wheat chromosomes were represented by the LGs, based on identity values higher than $80 \%$. The remaining LG was assigned to the chromosome arm 4DL.

Antibiosis to R. padi and plant pubescence

The phenotypic data for antibiosis did not diverge from a normal distribution according to the Anderson-Darling test $\left(A^{2}=0.33 ; p>0.25\right)$ but it appeared to be left-tailed $\left(k_{3}=-0.1\right)$ (Figure 1$)$. In chromosome 4BL we found one genomic region significantly associated to $R$. padi antibiosis. This region was flanked by the markers TP48882 and TP31989 within an interval of $4.9 \mathrm{cM}$ (Figure 2), and explained $10.2 \%$ of the total phenotypic variation (Table 1). Hereafter this locus will be referred to as $R p 1$. The closest marker TP48882 was $0.1 \mathrm{cM}$ away from $R p 1$. The heritability was $15.7 \%$ (Table 1 ).

The genomic region associated to pubescence was also located in chromosome 4BL, at a distance of $14.6 \mathrm{cM}$ from the antibiosis QTL. The pubescence screening showed $41.4 \%$ of the population to be pubescent and $58.6 \%$ glabrous, fitting a $1: 1$ segregation ratio $\left(d f=1 ; X^{2}=3.55 ; p=0.06\right)$, and indicating single gene inheritance. Groupings of RILs based on presence/absence of leaf pubescence showed no effect on $R$. padi antibiosis $(d f=1,135 ; F=0.323 ; p=$ 
0.574) or any other trait measured in this study. There were no epistatic interactions found in the two-dimensional scan.

Tolerance to $R$. padi

The phenotypic data for $R$. padi tolerance did not diverge from a normal distribution according to the AndersonDarling test $\left(A^{2}=0.45 ; p>0.25\right)$ but appeared to be right-tailed $\left(k_{3}=0.33\right)$ (Figure 1$)$. Two significant QTLs associated to $R$. padi tolerance were found, in chromosomes 5AL and 5BL, explaining 14.5 and $5.7 \%$ of the phenotypic variation, respectively (Table 2). The closest markers to these genomic regions are TP3728 and TP3351, respectively (Figure 2). Hereafter these loci will be referred to as $R p 2$ and $R p 3$, respectively. The heritability for this trait was $36.5 \%$.

The two-dimensional scan revealed one epistatic locus present in chromosome 3AL interacting with the marker TP3728 associated to Rp2 (Figure 3). The epistatic locus is closest to the marker TP5978, and herein referred as EnRp2. The interaction accounted for $5.0 \%$ of the phenotypic variation (Table 2). The percentage of the total phenotypic variation explained by the QTL model was $35.1 \%$.

The analysis of variance based on the marker classes showed that the interaction TP3728 * TP59798 was significant $(d f=1,81 ; F=16.26 ; p=0.0001$; Figure 4a), while there was no effect of TP59798 marker alone $(d f=1,81 ; F=$ $1.23 ; p=0.2721$ ). Figure $4 \mathrm{~b}$ shows the additive effect of the two loci $R p 2$ and $R p 3$.The comparison of means for all the combinations of these marker genotypes showed that the level of biomass reduction is lowest when $R p 2$ (TP3728) from the resistant parent is combined with the allele from the susceptible parent of marker TP59798 (Figure 5). The comparison of means also showed that TP59798 has no effect on $R$. padi tolerance (Figure 5).

The multi-environmental QTL analysis did not show genomic regions for plant growth per se to be associated to $R$. padi tolerance, and therefore, this data is not shown.

Resistance to S. graminum

The phenotypic data for number of S.graminum/tiller in the field did not diverge from a normal distribution according to the Anderson-Darling test $\left(A^{2}=0.51 ; p=0.193\right)$ but showed to be left-tailed $\left(k_{3}=-0.3\right)$ (Figure 1). Greenhouse phenotyping for $S$. graminum-induced chlorosis showed that $55.7 \%$ of the RILs were resistant and $44.3 \%$ were susceptible and this did not deviate from the expected segregation ratio of $1: 1\left(d f=1 ; X^{2}=1.82 ; p=\right.$ 0.17 ) for a single locus, most likely the previously mapped gene Gba by Zhu et al.(2005). The linkage analysis placed this locus in chromosome 7DL between markers TP81905 and TP13131 (Figure 2). Additionally, Gba was significantly associated to the number of $S$. graminum/tiller in the QTL analysis, explaining $16.6 \%$ of the phenotypic variation (Table 1).

Another genomic region, in chromosome 2DL, was also associated to the number of $S$. graminum/tiller, explaining $10.2 \%$ of the phenotypic variation (Table 1, Figure 2). The QTL model for aphids/tiller explained $29.1 \%$ of the total phenotypic variation, whereas the heritability was $57 \%$. The two-dimensional scan did not show any significant interaction between pairs of loci.

\section{DISCUSSION}

The next generation sequencing technologies provide large amounts of information and are suitable for identifying novel genomic regions associated to plant stresses, evolutionary studies and genome sequencing research. GBS in particular has proven to be very adequate for these tasks (Poland et al. 2012; Saintenac et al. 2013; Lu et al. 2013). Here we constructed GBS libraries and employed QTL analyses in an F6 RIL population to map known and novel genomic regions associated to resistance to two aphid species in wheat. 
We found one genomic region associated to $R$. padi antibiosis in chromosome 4BL (RpI), and a plant pubescence locus in the same chromosome arm. However, they were separated by a distance of $14.6 \mathrm{cM}$, and classification of RILs based on pubescence data showed no effect on antibiosis to R. padi. Some studies (Roberts and Foster 1983; Webster et al. 1994), but not all (Webster 1990; Papp and Mesterhazy 1993), have suggested that one possible cause for aphid resistance in wheat is the presence of trichomes in leaves. However, in our study neither $R$. padi nor $S$. graminum resistance was related to such a plant trait. This indicates that $R$. padi antibiosis and the number of $S$. graminum/tiller are caused by mechanisms different from pubescence in the resistant genotype CWI76364. So far, there are no other published reports that have investigated the genetic association between aphid resistance and plant pubescence in wheat. However, not only presence or absence of pubescence but also density and structure of the pubescence can be of importance for the resistance effect, as it has been shown in other plant species and insect herbivores, mainly chewers and miners (Dalin et al. 2008). We did not measure such characteristics in the pubescent genotypes since we found no differences in aphid resistance between glabrous and pubescent individuals.

It is probable that the pubescence locus we found is allelic to, or the same as the hairy leaf gene $H l l$ previously reported by Dobrovolskaya et al. (2007) in chromosome 4BL of Triticum dicoccoides L. However further evaluations are required to fully determine if this locus is the same as the one we found in Triticum dicoccum Schrank.

The genes $T b x 1$ and $T b x 2$ are other sets of genes previously discussed as resistance-related and found located in the proximal section of chromosome 4BL. These genes are involved in the synthesis of hydroxamic acids (hx) (Nomura et al. 2003). Those compounds have been reported to play an important role in the defense of gramineous plants when they are attacked by herbivores, causing antibiotic effects on insects including various aphid species (Frey et al. 1997; Niemeyer 2009). There are five hx-related genes in wheat that are identified as TaBxl through TaBx5 (Nomura et al. 2002; Nomura et al. 2003). These genes are located in chromosomes 4 and 5 of the three wheat genomes, and are involved in catalyzing the hydroxylation and ring expansion of indole-3-glycerol phosphate to synthesize DIBOA and DIMBOA (Frey et al. 1997; Nomura et al. 2003). Both DIBOA and DIMBOA are present in hexaploid wheat, however, only the latter appears to be present in high concentrations (Niemeyer et al. 1992). Niemeyer et al. (1992) found that the ancestor of A and D genomes of hexaploid wheat possesses low concentrations of hx, whereas Aegilops speltoides Tausch, the most likely donor of the B genome, and tetraploid wheat (AABB) possess high concentrations of DIMBOA, suggesting that the $\mathrm{B}$ genome is responsible for the main part of the synthesis of hx in hexaploid wheat. From our results is not possible determine whether Rpl is associated or not to Tbx genes, but it would be of great interest to analyze DIMBOA concentrations in the population.

Tolerance is an attractive component of insect resistance, as this trait does not pose any selection pressure on the insects and hence the risk of developing new virulent strains is absent compared to a scenario where insects are under constant selection due to antixenosis or antibiosis. In terms of crop management, tolerance may facilitate other aphid control methods, since when a control action is taken in commercial farms, aphids might already have built up their population levels above the economic threshold on a susceptible cultivar. A tolerant cultivar may endure higher aphid populations so there is more time for localizing the infestation and if necessary apply insecticides in the field.

Tolerant and susceptible plants differ in the quantity of biomass they produce under insect stress (Smith 2005). Causes for this can be due to differences in photosynthetic rate, allocation patterns and nutrient uptake (Rosenthal and Kotanen 1994). Since tolerance is related to various fundamental physiological mechanisms in plants, which are all influenced both by intrinsic and extrinsic factors, tolerance is a very complex and difficult trait to measure (Rosenthal and Kotanen 1994). Besides, it is often present along with antibiosis and antixenosis and it is also difficult to separate the tolerance effect from the others. Still, we were able to find genomic regions associated to wheat seedling tolerance to $R$. padi in chromosomes 5AL and 5BL ( $R p 2$ and $R p 3$, respectively). Since we measured tolerance to $R$. padi as biomass reduction, we considered the possibility that the accumulation of plant biomass per se may have contributed to tolerance, as plants with higher growth rates might have less biomass reduction due to aphid feeding. Therefore we conducted a multi-environmental QTL analysis to identify genomic regions associated to plant 
biomass per se, but we found that $R p 2$ and $R p 3$ were unrelated to it. This indicates that the regions we identified are only related to biomass reduction under $R$. padi stress and their expression might be induced by aphid feeding.

Since tolerance is a complex trait that involves several plant processes, its genetic effect can partly be explained by epistatic interactions. The implementation of the two-dimensional scan as described by Broman (2003), evaluates all pairwise interactions across the genome by comparing the log 10 likelihood ratio tests of two-QTL models with and without the epistatic term, as well as with the single-QTL models to finally obtain the LOD scores of such comparisons. The two-dimensional scan of $R$. padi tolerance displayed a significant epistatic interaction that changes the magnitude of $R p 2$ caused by a genomic region located in 3AL, linked to the marker TP59798 (EnRp2). Tolerance to $R$. padi appears to be enhanced when the marker allele of TP59798 from the susceptible parent Seri M82 is present along with $R p 2$. The epistatic locus EnRp2 had in itself no significant effect on biomass reduction due to R. padi feeding. According to Mackay (2013) the conditions for identification of epistatic interaction are optimal in biparental mapping populations because allele frequencies tend to be equal to 0.5 and thus the epistatic variance is maximized. On the other hand, the effect of the interactions can be significant even though the interacting loci have no significant effect individually (Mackay 2013).

Evaluation of tolerance to $R$. padi is difficult since the absence of visual symptoms requires the measurement of plant growth under aphid-infested and non-infested treatments. Consequently the phenotyping entails stringent conditions, such as to guarantee that each plant genotype in the pair of treatments have approximately the same starting size, something which can be highly influenced by seed quality and growing conditions. Additionally, to rule out antibiosis and/or antixenosis effects is important to ensure that plants have approximately the same aphid density over time. To meet these requirements in the evaluations we selected pairs of seedlings with approximately the same size and periodically infested with aphids to keep approximately the same aphid density throughout the phenotyping process. However, once proven to be of significant importance for biomass and eventually seed production, molecular markers such as those provided by our study allow marker-based selection in wheat breeding material without all the efforts needed to phenotype segregating populations and careful control of test conditions for phenotyping as described above.

There are 14 previously reported $S$. graminum resistance genes in wheat and wheat relatives, and nine of those have been found in the chromosome 7DL from A. tauschii (McIntosh et al. 2010). However, six of the nine genes reported in 7DL may be allelic or closely linked to $G b 3$ (Zhu et al. 2005). Among those, the Gba gene originates from the same A. tauschii (1027) accession used in the development of CWI76364. In the present study we were able to confirm this resistance locus in chromosome 7DL by sequenced-based genotyping.

In a microarray study comparing plants with and without $S$. graminum, Reddy et al. (2013) showed that aphidinfested plants carrying $G b 3$ gene prevent cell wall modification and consequently cell death, and also down-regulate genes for the synthesis of secondary metabolites related to plant defenses. Most probably the tolerance component of $G b a$ plants is explained by similar molecular mechanisms. Resistance characterization of the $G b 3$ gene has shown that it also confers both antixenotic and antibiotic effect on biotype E of S. graminum (Weng et al. 2004). This may explain the lower number of aphids/tiller that we found in the field, in our case caused by Gba. This makes the Gba gene a good candidate to be deployed in elite wheat germplasm.

Additionally, we found chromosome 2DL to be a novel region associated to the frequency of $S$. graminum/tiller in the field. This QTL, here referred to as $Q G b 8$, contributed $10.2 \%$ of the phenotypic variation. We attribute that the 2DL region was not previously reported in A. tauschii (1027) because it was associated to aphids/tiller in the field, whereas Zhu et al. (2005) evaluated symptoms based on chlorosis (tolerance) in a greenhouse test similar to ours, where we also did not find any association to 2DL. It is possible that $Q G b 8$ has a predominantly antixenotic effect on S. graminum since Lage et al. (Lage et al. 2003) showed that A. tauschii accession\#1027 was the most antixenotic among 12 studied SHWs. However, reduced aphids/tiller could also be due to antibiosis since CWI76364 showed to be more antixenotic, antibiotic and tolerant than the susceptible cultivar Seri M82 (Lage et al. 2003). Further 
investigation is required to confirm and characterize $Q G b 8$ and determine the type of resistance it confers against $S$. graminum.

Another possible explanation for the new QTL for S. graminum resistance might be that there was an uncharacterized biotype of this aphid in our field. We did not try to determine the biotypes of S. graminum, however Burd and Porter (2006) reported that biotype E and I are the most common in wheat in USA.

All phenotypic responses fitted a normal distribution with some degree of skewness, possibly because the environmental variance was relatively large compared to the genetic variance (Lynch and Walsh 1998). This is particularly the case for $R$. padi antibiosis where we observed a low heritability estimate. However, low heritability seems to be a common feature of insect life history traits, since they are highly influenced by the environment (Price and Schluter 1991).

In conclusion, we were able to utilize a sequence-based genotyping method to determine the genetic bases of resistance to $R$. padi and $S$. graminum in the synthetic hexaploid wheat CWI76364. Derived from this, we found one locus for $R$. padi antibiosis (Rpl) in chromosome 4BL and two loci for $R$. padi tolerance $(R p 2$ and $R p 3)$ in chromosomes $5 \mathrm{AL}$ and $5 \mathrm{BL}$, respectively. We also found one locus that originates from the susceptible parent (EnRp2) that enhances the effect of $R p 2$. From these results we can also conclude that resistance to $R$. padi originates from $T$. dicoccum in the CWI76364/Seri M82 population. This is the first report on the genetic mapping of antibiosis, tolerance and epistatic effects against $R$. padi in hexaploid wheat. Using GBS, we were able to re-map the Gba $S$. graminum resistance gene, and were also able to find in chromosome 2DL a genomic region $(Q G b 8)$ associated to the number of $S$. graminum/tiller in the field. Plant pubescence was not related to any of the resistance traits we measured for any of the aphid species. The identification of the GBS markers associated with the resistance loci will help to fine map those genomic regions and to develop new molecular markers that are easier to apply in wheat breeding. We think that the loci we found are promising sources to be deployed in elite wheat gemplasm, since several resistance mechanisms should make the resistance more durable. Nonetheless, more efforts in finding and characterizing additional sources of resistance are needed in order to further enhance wheat cultivars' resistance to $S$. graminum and $R$. padi. By increasing the knowledge in this area it will be possible to diminish the use of pesticides and consequently it will contribute to a more environment friendly production of wheat.

Acknowledgments

We thank the Monsanto's Beachell-Borlaug International Scholars Program for financial support. We also thank Stelle Proux-Wéra (SLU) for bioinformatic support through the PlantLink Network (SLU). We thank Jorge Montoya (CIMMYT) for assistance in conducting the field trial. 


\section{REFERENCES}

Berzonsky WA, Ding H, Haley SD, Harris MO, Lamb RJ, McKenzie RIH, Ohm HW, Patterson FL, Peairs FB, Porter DR, Ratcliffe RH, Shanower TG (2003) Breeding wheat for resistance to insects. Plant Breeding Reviews 22:221-296

Blackman RL, Eastop VF (2007) Taxonomic Issues. In: Van Emden HF, R. H (eds) Aphids as Crop Pests. CAB International, Oxfordshire, UK, pp 1-29

Brenchley R, Spannagl M, Pfeifer M, Barker GL, D’Amore R, Allen AM, McKenzie N, Kramer M, Kerhornou A, Bolser D (2012) Analysis of the bread wheat genome using whole-genome shotgun sequencing. Nature 491 (7426):705-710

Broman KW, Wu H, Sen Ś, Churchill GA (2003) R/qtl: QTL mapping in experimental crosses. Bioinformatics 19 (7):889-890

Burd JD, Porter DR (2006) Biotypic diversity in greenbug (Hemiptera: Aphididae): characterizing new virulence and host associations. J Econ Entomol 99 (3):959-965

Cheung WY, Di Giorgio L, Ahman I (2010) Mapping resistance to the bird cherry-oat aphid (Rhopalosiphum padi) in barley. Plant Breeding 129 (6):637-646. doi:10.1111/j.1439-0523.2010.01771.x

Crespo-Herrera LA (2012) Resistance to aphids in wheat: from a plant breeding perspective. Introductory paper at the Faculty of Landscape Planning, Horticulture and Agricultural Science. Swedish University of Agricultural Sciences, Alnarp, Sweden

Da Costa E Silva L, Wang S, Zeng Z-B (2012a) Multiple trait multiple interval mapping of quantitative trait loci from inbred line crosses. BMC genetics 13 (1):67

Da Costa E Silva L, Wang S, Zeng ZB (2012b) Composite interval mapping and multiple interval mapping: procedures and guidelines for using windows QTL Cartographer. In: Rifkin SA (ed) Quantitative Trait Loci (QTL): Methods and Protocols. Methods in molecular biology. Humana Press, New York, US, pp 75-119

Dalin P, Ågren J, Björkman C, Huttunen P, K K (2008) Leaf trichome formation and plant resistance to herbivore. In: Schaller A (ed) Induced plant resistance to herbivore. Springer, Amsterdam, Netherlands, pp 89-105

Dobrovolskaya O, Pshenichnikova T, Arbuzova V, Lohwasser U, Röder M, Börner A (2007) Molecular mapping of genes determining hairy leaf character in common wheat with respect to other species of the Triticeae. Euphytica 155 (3):285-293

Dunn BL, Carver BF, Baker CA, Porter DR (2007) Rapid phenotypic assessment of bird cherry-oat aphid resistance in winter wheat. Plant Breeding 126 (3):240-243. doi:10.1111/j.1439-0523.2007.01345.x

Elshire RJ, Glaubitz JC, Sun Q, Poland JA, Kawamoto K, Buckler ES, Mitchell SE (2011) A Robust, Simple Genotyping-by-Sequencing (GBS) Approach for High Diversity Species. PLoS ONE 6 (5):e19379. doi:10.1371/journal.pone.0019379

Frey M, Chomet P, Glawischnig E, Stettner C, Grün S, Winklmair A, Eisenreich W, Bacher A, Meeley RB, Briggs SP, Simcox K, Gierl A (1997) Analysis of a chemical plant defense mechanism in grasses. Science 277 (5326):696-699. doi:10.1126/science.277.5326.696

Kieckhefer RW, Gellner JL (1992) Yield losses in winter-wheat caused by low-density cereal aphid populations. Agron J 84 (2):180-183

Lage J, Skovmand B, Andersen SB (2003) Characterization of greenbug (Homoptera : Aphididae) resistance in synthetic hexaploid wheats. J Econ Entomol 96 (6):1922-1928

Li H, Ye G, Wang J (2007) A modified algorithm for the improvement of composite interval mapping. Genetics 175 (1):361-374

Lu F, Lipka AE, Glaubitz J, Elshire R, Cherney JH, Casler MD, Buckler ES, Costich DE (2013) Switchgrass Genomic Diversity, Ploidy, and Evolution: Novel Insights from a Network-Based SNP Discovery Protocol. PLoS Genet 9 (1):e1003215. doi:10.1371/journal.pgen.1003215

Lynch M, Walsh B (1998) Genetics and analysis of quantitative traits.

Mackay TF (2013) Epistasis and quantitative traits: using model organisms to study gene-gene interactions. Nature Reviews Genetics

McIntosh RA, Yamazaki Y, Dubcovsky J, Rogers J, Morris C, Somers DJ, Appels R, Devos KM (2010) Catalogue of Gene Symbols for Wheat. 2011 edn. http://www.shigen.nig.ac.jp/wheat/komugi/,

Niemeyer HM (2009) Hydroxamic Acids Derived from 2-Hydroxy-2H-1,4-Benzoxazin-3(4H)-one: Key Defense Chemicals of Cereals. J Agric Food Chem 57 (5):1677-1696. doi:10.1021/jf8034034

Niemeyer HM, Copaja SV, Barria BN (1992) The Triticeae as sources of hydroxamic acids, secondary metabolites in wheat conferring resistance against aphids. Hereditas 116 (3):295-299. doi:10.1111/j.16015223.1992.tb00158.x 
Nomura T, Ishihara A, Imaishi H, Endo T, Ohkawa H, Iwamura H (2002) Molecular characterization and chromosomal localization of cytochrome $\mathrm{P} 450$ genes involved in the biosynthesis of cyclic hydroxamic acids in hexaploid wheat. Mol Genet Genomics 267 (2):210-217. doi:10.1007/s00438-002-0653-x

Nomura T, Ishihara A, Imaishi H, Ohkawa H, Endo T, Iwamura H (2003) Rearrangement of the genes for the biosynthesis of benzoxazinones in the evolution of Triticeae species. Planta 217 (5):776-782. doi:10.1007/s00425-003-1040-5

Ogbonnaya FC, Abdalla O, Mujeeb-Kazi A, Kazi AG, Xu SS, Gosman N, Lagudah ES, Bonnett D, Sorrells ME, Tsujimoto H (2013) Synthetic hexaploids: harnessing species of the primary gene pool for wheat improvement. Plant Breeding Reviews, Volume 37:35-122

Papp M, Mesterhazy A (1993) Resistance to bird cherry-oat aphid (Rhopalosiphum padi L.) in winter wheat varieties. Euphytica 67 (1-2):49-57

Poland JA, Brown PJ, Sorrells ME, Jannink J-L (2012) Development of High-Density Genetic Maps for Barley and Wheat Using a Novel Two-Enzyme Genotyping-by-Sequencing Approach. PLoS ONE 7 (2):e32253. doi:10.1371/journal.pone.0032253

Porter DR, Burd JD, Shufran KA, Webster JA, Teetes GL (1997) Greenbug (Homoptera: Aphididae) biotypes: selected by resistant cultivars or preadapted opportunists? J Econ Entomol 90 (5):1055-1065

Price T, Schluter D (1991) On the low heritability of life-history traits. Evolution 45 (4):853-861

$\mathrm{R}$ core team (2013) R: a language and environment for statistical computing. Vienna, Austria

Reddy SK, Weng Y, Rudd JC, Akhunova A, Liu S (2013) Transcriptomics of induced defense responses to greenbug aphid feeding in near isogenic wheat lines. Plant Sci 212:26-36

Riedell WE, Kieckhefer RW, Langham MAC, Hesler LS (2003) Root and shoot responses to bird cherry-oat aphids and barley yellow dwarf virus in spring wheat. Crop Sci 43 (4):1380-1386

Roberts JJ, Foster JE (1983) Effect of leaf pubescence in wheat on the bird cherry-oat aphid (Homoptera: Aphididae). J Econ Entomol 76 (6):1320-1322

Rosenthal J, Kotanen P (1994) Terrestrial plant tolerance to herbivory. Trends Ecol Evol 9 (4):145-148

Saintenac C, Zhang D, Wang S, Akhunov E (2013) Sequence-Based Mapping of the Polyploid Wheat Genome. G3: Genes|Genomes|Genetics. doi:10.1534/g3.113.005819

SAS Institute Inc (2011) SAS/STAT ${ }^{\circledR} 9.3$ User's guide. SAS Institute Inc, Cary, NC, USA

Smith CM (2005) Plant Resistance to Arthropods: Molecular and Conventional Approaches. Springer, Aa Dordrecht, Netherlands

Smith CM, Starkey S (2003) Resistance to greenbug (Heteroptera : Aphididae) biotype I in Aegilops tauschii synthetic wheats. J Econ Entomol 96 (5):1571-1576. doi:10.1603/0022-0493-96.5.1571

Spindel J, Wright M, Chen C, Cobb J, Gage J, Harrington S, Lorieux M, Ahmadi N, McCouch S (2013) Bridging the genotyping gap: using genotyping by sequencing (GBS) to add high-density SNP markers and new value to traditional bi-parental mapping and breeding populations. Theor Appl Genet 126 (11):2699-2716. doi:10.1007/s00122-013-2166-x

Voorrips R (2002) MapChart: software for the graphical presentation of linkage maps and QTLs. Journal of Heredity 93 (1):77-78

Voss TS, Kieckhefer RW, Fuller BW, McLeod MJ, Beck DA (1997) Yield losses in maturing spring wheat caused by cereal aphids (Homoptera: Aphididae) under laboratory conditions. J Econ Entomol 90 (5):1346-1350

Webster J (1990) Resistance in triticale to the Russian wheat aphid (Homoptera: Aphididae). J Econ Entomol 83 (3):1091-1095

Webster J, Inayatullah C, Hamissou M, Mirkes K (1994) Leaf pubescence effects in wheat on yellow sugarcane aphids and greenbugs (Homoptera: Aphididae). J Econ Entomol 87 (1):231-240

Weng Y, Lazar MD, Michels GJ, Rudd JC (2004) Phenotypic mechanisms of host resistance against greenbug (Homoptera : Aphididae) revealed by near isoaenic lines of wheat. J Econ Entomol 97 (2):654-660

Zhu LC, Smith CM, Fritz A, Boyko E, Voothuluru P, Gill BS (2005) Inheritance and molecular mapping of new greenbug resistance genes in wheat germplasms derived from Aegilops tauschii. Theor Appl Genet 111 (5):831-837. doi:10.1007/s00122-005-0003-6 
Table 1. Main QTL location, LOD scores, additive effects and heritability for Rhopalosiphum padi antibiosis and Schizaphis graminum aphids/tiller.

\begin{tabular}{|c|c|c|c|c|c|c|c|c|}
\hline QTL & Trait & Flanking markers & $\mathrm{LG}^{\dagger}$ & QTL interval (cM) & LOD score & Effect & $\mathrm{PVE}^{\star}(\%)$ by QTL & Heritability \\
\hline$R p 1$ & R. padi-antibiosis & TP48882; TP31989 & $4 \mathrm{BL}$ & $31.1-36.0$ & 3.1 & -5.2 & 10.2 & 15.7 \\
\hline Gba & S. graminum/tiller & ТР81905; Gba & 7DL & 116.1-119.8 & 5.9 & -0.1 & 16.6 & 57.0 \\
\hline$Q G b 8$ & $? ? ?$ & ТР67214; ТР84201 & $2 \mathrm{DL}$ & $71.7-74.8$ & 3.9 & -0.1 & 10.2 & \\
\hline
\end{tabular}

${ }^{\ddagger} \mathrm{LG}=$ Linkage group; ${ }^{\ddagger}$ Proportion of the total phenotypic variance explained by the QTL

Table 2. Main QTL location, LOD scores, additive effects, interaction effect and heritability for Rhopalosiphum padi tolerance.

\begin{tabular}{|c|c|c|c|c|c|c|c|}
\hline QTL & Flanking markers & $\mathrm{LG} \dagger$ & Interval (cM) & LOD & Effect & PVE $\ddagger(\%)$ by QTL & Heritability \\
\hline$R p 2$ & TP3728; TP38148 & $5 \mathrm{AL}$ & $106.5-107.0$ & 3.7 & -2.4 & 14.5 & 36.51 \\
\hline Rp3 & TP3351; TP17691 & $5 \mathrm{BL}$ & $143.2-148.9$ & 3.8 & -2.7 & 5.7 & \\
\hline EnRp2 & ТР62232;ТР59798 & $3 \mathrm{AL}$ & $99.7-100.2$ & 0.35 & 0.6 & 6.7 & \\
\hline$R p 2 * E n R p 2$ & ТР3728*ТР59798 & & & 3.1 & 1.6 & 5.0 & \\
\hline
\end{tabular}

${ }^{\dagger} \mathrm{LG}=$ Linkage group; ${ }^{\dagger}$ Proportion of the total phenotypic variance explained by the QTL 

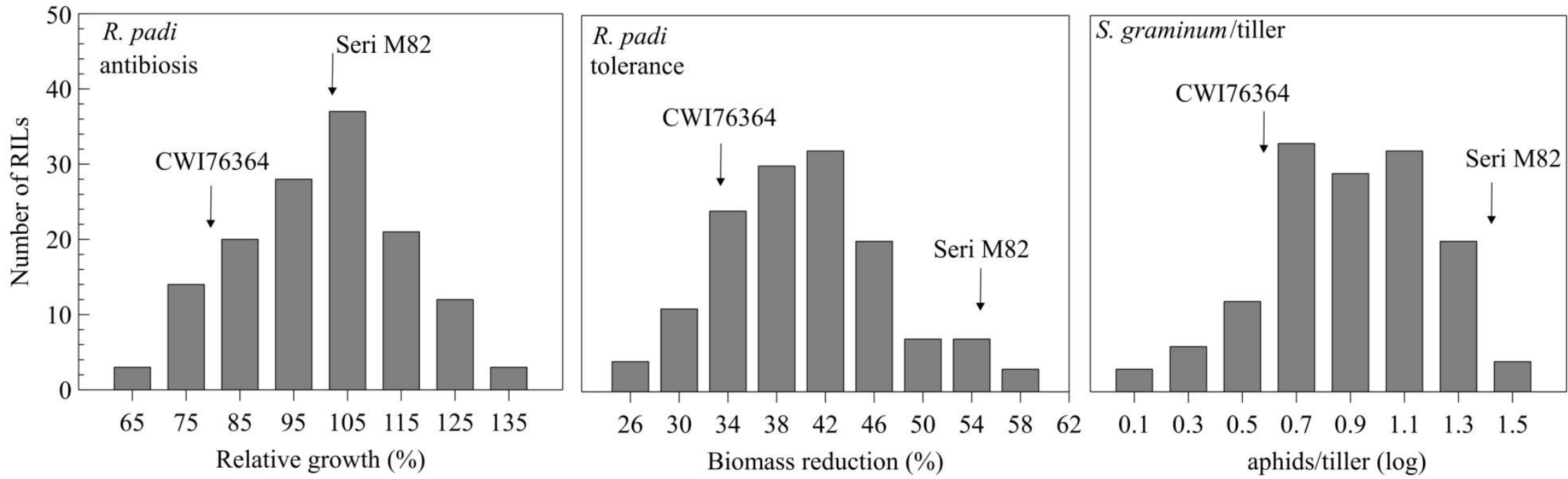

Figure 1. Histograms of the phenotypic response of the RILs to R. padi antibiosis, R. padi tolerance and S. graminum/tiller. The arrows indicate the phenotypic value of the two parents of the population. 

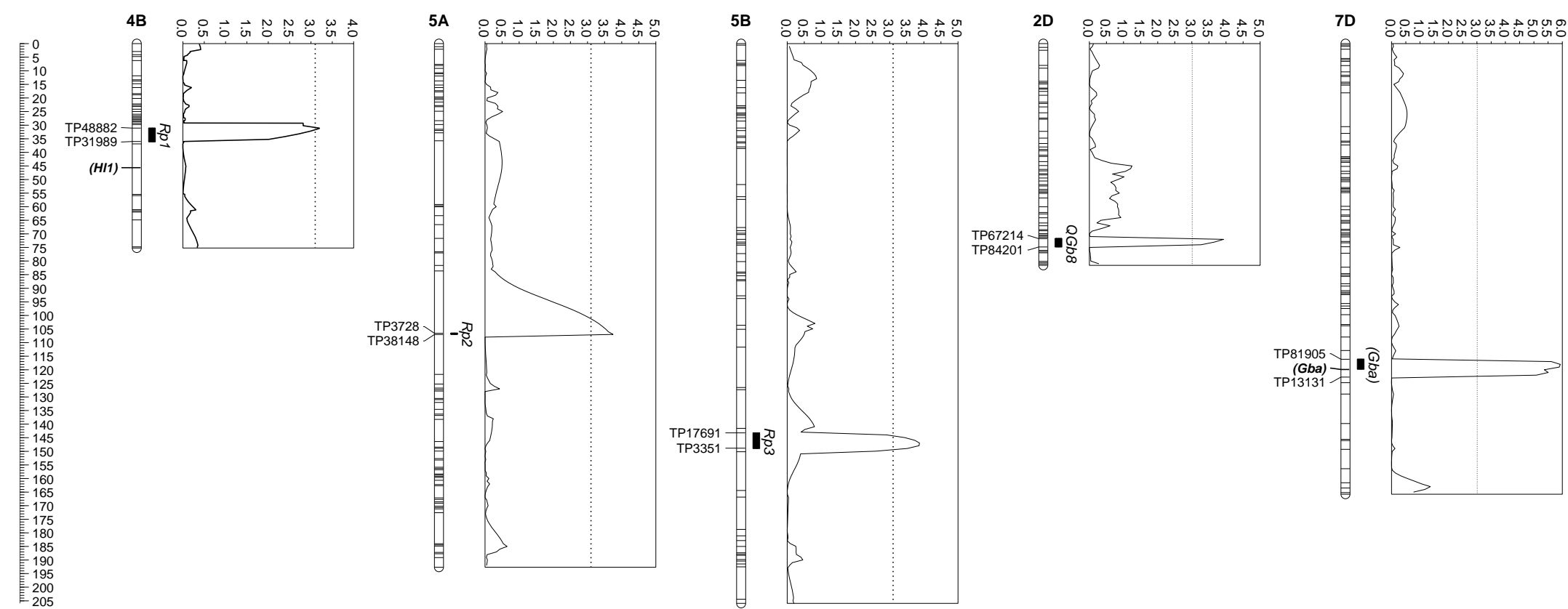

Figure 2. Linkage maps and LOD profiles of genomic regions associated to $R$. padi and $S$. graminum resistance in chromosomes 4B, 5A, 5B, 2D and 7D. The loci inside the parentheses indicate the putative genes. 


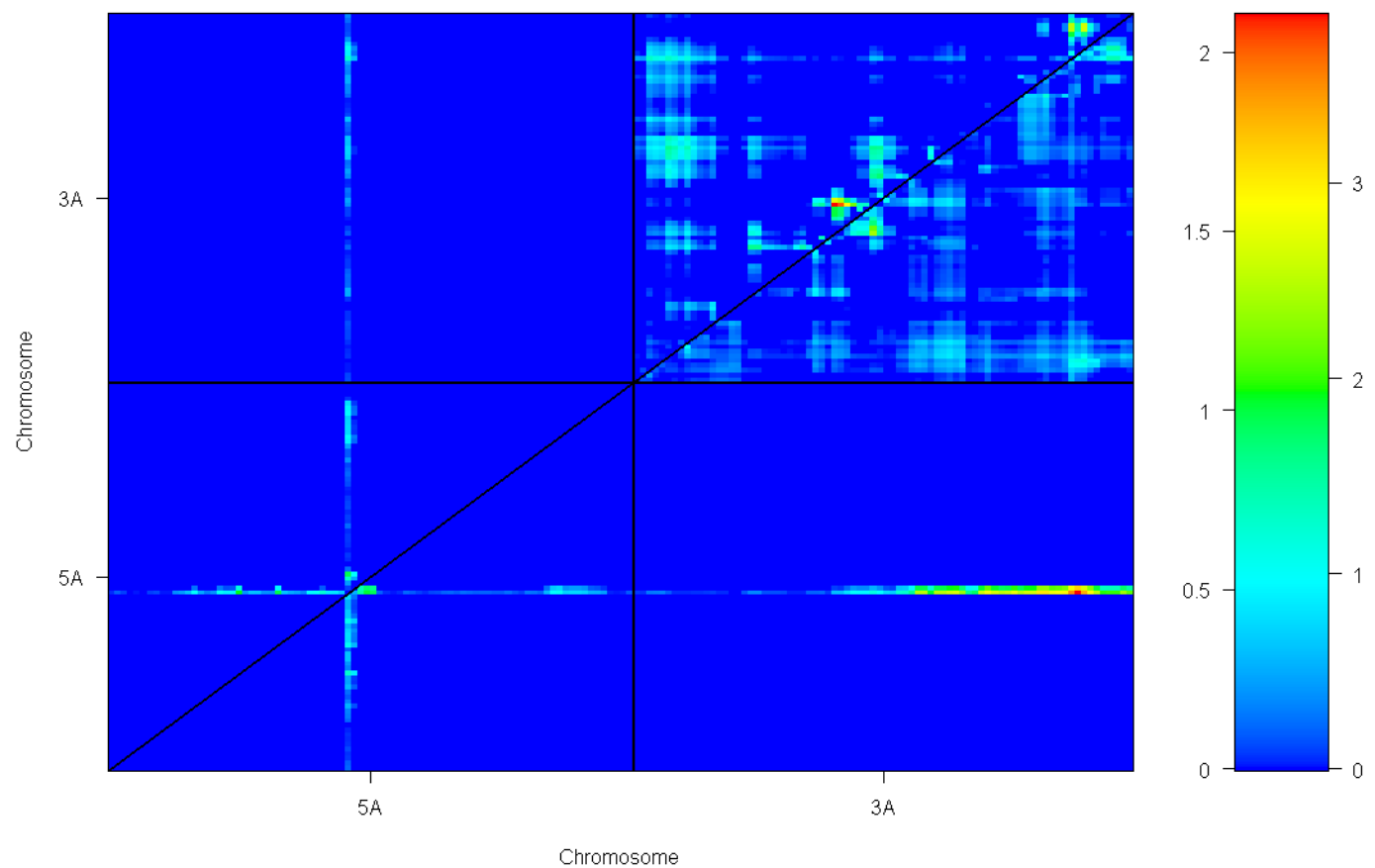

Figure 3. Heat plot of the 2-dimensional 2-qtl scan of chromosomes 5A and 3A. The triangle above the diagonal displays the LOD score of the model assuming only additive effects. The triangle beneath the diagonal displays the LOD scores of the model assuming that there is epistatic interaction between loci. 

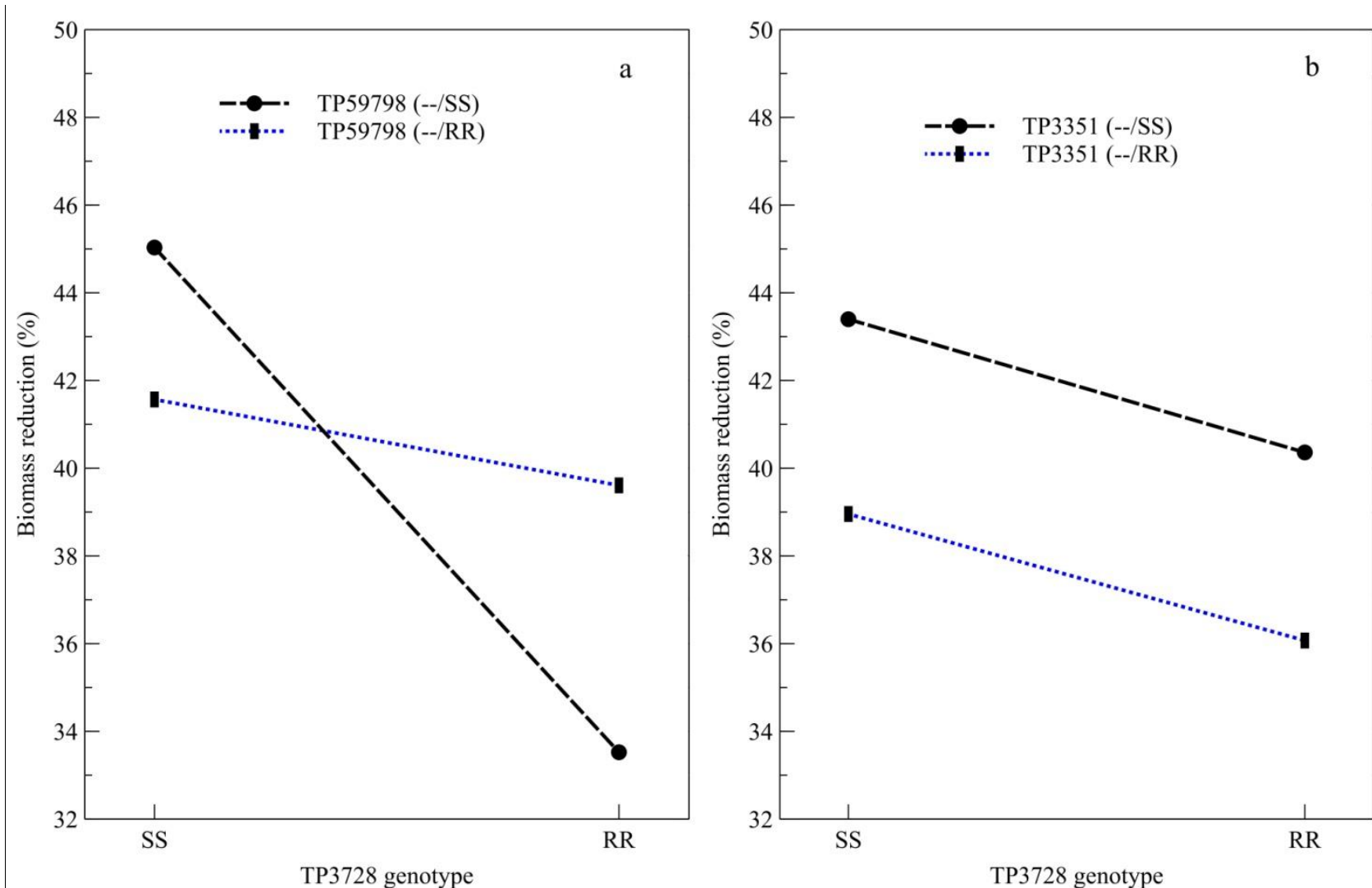

Figure 4. Interaction plots between, a) marker TP3728 linked to a main QTL in chromosome 5A and the epistatic locus linked to marker TP5978 in chromosome 3A, and b) Markers linked to main QTL effect displaying additive effect. Marker codes indicate whether alleles originate from the susceptible (SS) or resistant (RR) parent. 


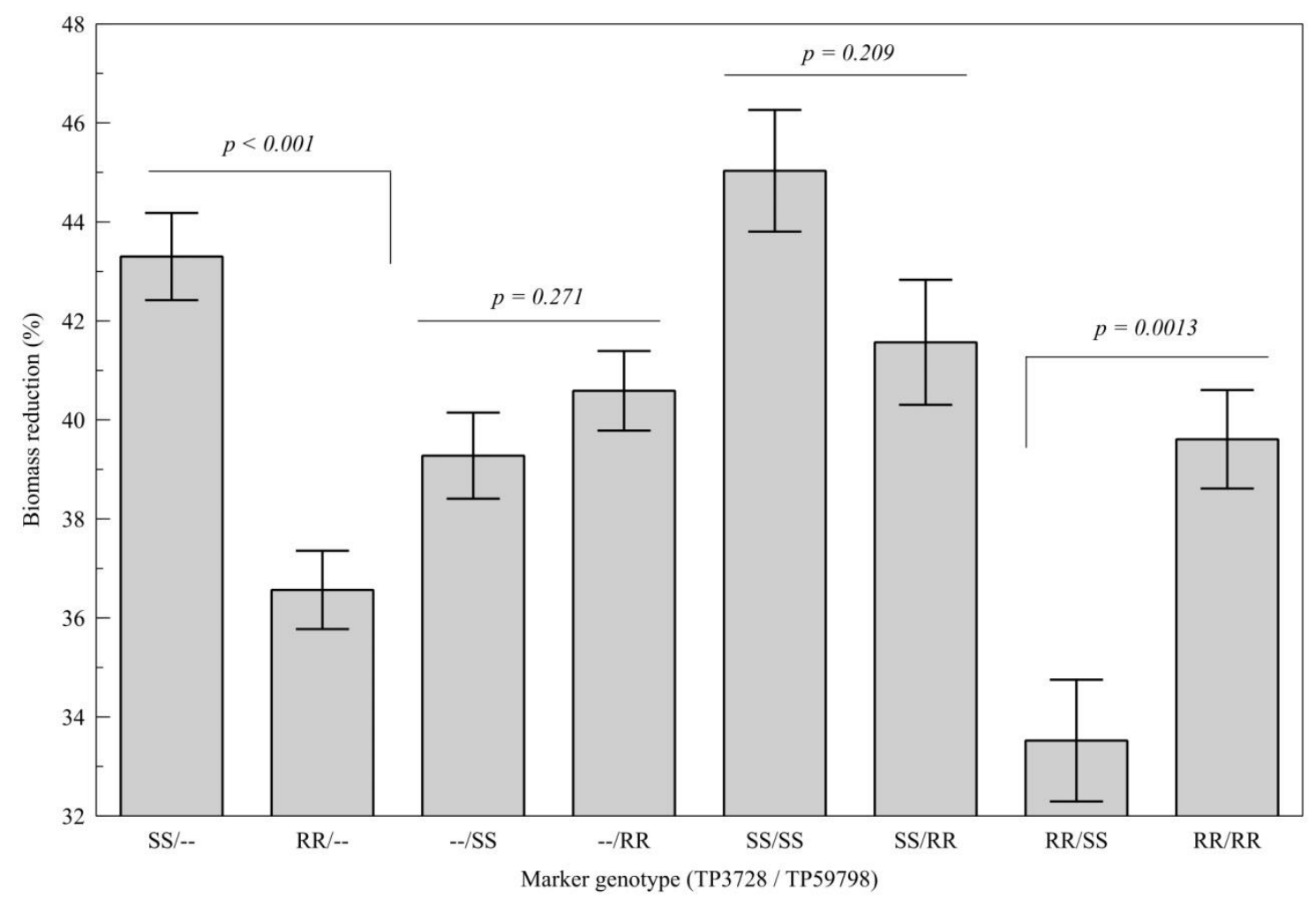

Figure 5. Phenotypic means and standard errors of markers linked to interacting loci associated the biomass reduction due to R. padi feeding. Marker codes indicate whether alleles originate from the susceptible (SS) or resistant (RR) parent. The $p$-values above the lines indicate the significance level in Tukey's tests for the pairs of means beneath the lines. 